\title{
Computer assisted semen analysis for quantification of motion characteristics of bull sperm during cryopreservation cycle
}

\author{
M. N. Sundararaman, J Kalatharan, K Thilak Pon Jawahar \\ Frozen Semen Bank, Department of Animal Genetics and Breeding \\ Tamil Nadu Veterinary and Animal Sciences University, Madras Veterinary College, Chennai - 600007, India \\ Corresponding author: M. N. Sundararaman, email: yemenyes@yahoo.com \\ Received: 09-05-2012, Accepted: 05-06-2012, Published Online: 01-11-2012 \\ doi: $10.5455 /$ vetworld.2012.723-726
}

\begin{abstract}
Aim: The study was undertaken to quantify and to analyze the changes in the motion characteristics of bull spermatozoa during various stages of cryopreservation cycle.

Materials and Methods: Using computer assisted semen analysis (CASA) technique, 26 ejaculates, collected from two Jersey bulls were analyzed for motility, head behaviour and swimming pattern of spermatozoa on dilution, pre-freeze and post-thaw stages of cryopreservation. French straw technique was employed for deep-freezing of semen using liquid nitrogen. Results: Equilibration of diluted semen at $5^{\circ} \mathrm{C}$ has significantly $(\mathrm{P} \leq 0.01)$ reduced sperm motility, progressive motility, path velocity, and progressive velocity. Beat cross frequency was also affected significantly $(\mathrm{P} \leq 0.05)$ by equilibration. Freezing and thawing processes drastically affected all the motility, velocity and head behaviour characteristics $(\mathrm{P}<0.01)$.

Conclusion: CASA facilitate objective evaluation sperm motion characteristics. Adoption of CASA technique has the potential for improvements in evaluation of semen thereby the quality of frozen semen for fertility can be enhanced.

Key words: bull, cryopreservation, CASA, spermatozoa,

To cite this article: Sundararaman MN, Kalatharan J, Jawahar KTP (2012) Computer assisted semen analysis for quantification of motion characteristics of bull sperm during cryopreservation cycle, Vet World, 5(12): 723-726, doi: 10.5455/vetworld.2012.723-726
\end{abstract}

\section{I ntroduction}

Artificial insemination (AI) in bovines is a classical example for successful application of a biotechnological method at field level. Although fertility of frozen-thawed semen is generally acceptable but the efficiency of cryopreservation is still relatively low. Rapid rate of cryopreservation results in cell damage to spermatozoa due to osmotic imbalance [1].

The mammalian spermatozoa acquire the capacity for flagellar movements during epididymal transport and it is modulated in the female reproductive tract before termination during fertilization. These changes in flagellar activity reflect the physiological events within the sperm cell including the metabolic activity and alteration of the sperm membrane [2]. Further the events of semen processing during cryopreservation cycle bring injuries to sperm membrane leading to changes in the sperm physiology which may be reflected in sperm motility [3]. Cryopreservation also affects the functional integrity of acrosome and mitochondria that is responsible for the generation of energy from intracellular stores of ATP [4].

Conventionally, sperm motility estimation is done by visual approximation of progressively moving spermatozoa using phase contrast microscope. The progressive motility estimation is only an assessment of 'quantity' of moving spermatozoa. However, the assessment of quality of motility in terms of velocity, swimming pattern, sperm head behaviour etc., may help in better understanding of the possible sperm function. The advent of computer assisted semen analysis (CASA) has brought a new dimension to semen evaluation. The CASA technique yields repeatable and highly reliable results on kinematics of ejaculates based on measurements of individual sperm cells. Therefore, assessment of sperm motility coupled with kinetic measurements would help in better evaluation of semen quality.

\section{Materials and Methods}

Two Jersey bulls, aged 2 years, maintained for instructional purpose for the students of Madras Veterinary College, Chennai under stall-fed conditions were used for the study. The bulls were fed with $3 \mathrm{~kg}$ of concentrate/day/bull in addition to $3 \mathrm{~kg}$ of dry fodder per bull. Water and green fodder were provided ad libitum. Since semen collection by artificial vagina method did not involve any intervention in the normal physiology of the animals, this experiment was exempted approval from Institutional Animal Ethics 
Table-1. Analysis set-up for HT-IVOS version 10.9 used to evaluate bull spermatozoa

\begin{tabular}{lc}
\hline Variables & Settings \\
\hline Frame rate (Hz) & 60 \\
Frames acquired & 30 \\
Minimum contrast & 40 \\
Minimum cell size (pixels) & 8 \\
Threshold straightness (\%) & 64 \\
Medium VAP cut-off (m/s) & 80 \\
Low VAP cut-off (m/s) & 15 \\
Low VSL cut-off (m/s) & 4.4 \\
Non-motile head intensity & 80 \\
Static size limit - minimum & 0.38 \\
Static size limit - maximum & 1.49 \\
Static intensity limit - minimum & 0.42 \\
Static intensity limit - maximum & 1.35 \\
Static elongation limit - minimum & 12 \\
Static elongation limit - maximum & 81 \\
Optic calibration & \\
Magnification & 1.89 \\
Camera frequency (Hz) & 60 \\
Stage configuration - Makler & 10 \\
Chamber depth (m) & 16.3 \\
Motile position (mm) & 16.3 \\
Static position (mm) &
\end{tabular}

Committee of Tamil Nadu Veterinary and Animal Sciences University, Chennai, India.

Semen was collected using artificial vagina. Two successive ejaculates were taken in a collection schedule. A total of 26 ejaculates, 14 and 12 from the 2 bulls respectively were utilized for the study. The semen samples were evaluated for ejaculate volume, sperm concentration and sperm motility by conventional methods. Only samples having progressive sperm motility of $70 \%$ and above by manual evaluation were included in the study. Tris buffer based diluent, having $20 \%$ egg yolk and $7 \%$ glycerol was used to extend the semen so as to fix the sperm number as 30 million per insemination dose. Later, semen samples were filled and sealed in $0.25 \mathrm{ml}$ French medium straws using automatic filling and sealing machine. The diluted semen samples were equilibrated for 4 hours at $5 \mathrm{C}$ in a cold handling cabinet.

By exposing the straws horizontally to liquid nitrogen vapour for 15 minutes the semen was frozen and immersed in liquid nitrogen for final freezing and storage at $-196^{\circ} \mathrm{C}$.

An aliquot of the extended semen was further diluted for the CASA analysis by adjusting the sperm concentration to $20 \times 10^{6} / \mathrm{ml}$ using Tris buffer. CASA analysis was done by using Hamilton Thorne integrated visual optical system (IVOS) version 10.9 (Table-1). The chamber temperature was set at $37^{\circ} \mathrm{C}$. One microlitre of the prepared semen sample was loaded on the Makler counting chamber (Self-Medical Ints., Ltd.) and cover glass was placed on the droplet. Ten microscopic fields were analyzed for each semen sample. The CASA analysis of the semen was done on dilution and after equilibration (pre-freeze). After 24 hours of storage, the cryopreserved semen samples were thawed in a water bath at $37^{\circ} \mathrm{C}$ for 30 seconds and the CASA analysis was done (post-thaw) by repeating the procedure adopted for pre-freeze semen.

During the analysis, the sperm motion characteristics like sperm motility (SM) (\%), progressive motility (PSM) (\%), path velocity (VAP) (m/s), progressive velocity (VSL) $(\mathrm{m} / \mathrm{s})$, curvilinear velocity (VCL) $(\mathrm{m} / \mathrm{s})$, lateral amplitude of head displacement $(\mathrm{ALH})(\mathrm{m})$, beat cross frequency $(\mathrm{Hz})$ and straightness (STR) $(\%)$ were studied.

The data obtained was analyzed by one-way analysis of variance using microstat software (Ecosof Inc., 1984, Baltimore, USA) at $\mathrm{P} \leq 0.05$ and $\mathrm{P} \leq 0.01$ level of significance.

\section{Results}

The motility characteristics of bull spermatozoa, SM and PSM showed highly significant difference (P $\leq 0.01$ ) among the dilution, pre-freeze and post-thaw stages of cryopreservation cycle (Table-2).

Among the velocity traits, the variations among the stages of cryopreservation cycle were highly significant ( $\mathrm{P} \leq 0.01$ ) for VAP and VSL (Table-2) and was significant $(\mathrm{P} \leq 0.05)$ for $\mathrm{VCL}$ between pre-freeze and post-thaw and between the stages of dilution and pre-freeze.

The results pertaining to ALH also exhibited highly significant $(\mathrm{P} \leq 0.01)$ decline from pre-freeze to post-thaw stage, while the difference between the stage of dilution and pre-freeze was not significant (Table-2). Highly significant $(\mathrm{P} \leq 0.01)$ variations were seen in BCF of bull spermatozoa among the different 
Table-2. Means for motion characteristics of bull spermatozoa during cryopreservation cycle

\begin{tabular}{|c|c|c|c|c|c|}
\hline Sr. No. & Sperm Motion Characteristics & $\begin{array}{r}\text { Stages } \\
\text { On dilution }\end{array}$ & $\begin{array}{l}\text { f Cryoprese } \\
\text { Pre-freeze }\end{array}$ & $\begin{array}{l}\text { ation } \\
\text { Post-thaw }\end{array}$ & Level of Significance \\
\hline 1 & Sperm motility (SM) (\%) & $94.3^{\mathrm{a}}$ & $89.4^{\mathrm{b}}$ & $63.0^{\mathrm{c}}$ & $P \leq 0.01$ \\
\hline 3 & Path velocity $(\mathrm{VAP})(\mathrm{m} / \mathrm{s})$ & $117.4^{\mathrm{a}}$ & $110.6^{b}$ & $90.7^{c}$ & $P \leq 0.01$ \\
\hline 4 & Progressive velocity (VSL) $(\mathrm{m} / \mathrm{s})$ & $94.1^{\mathrm{a}}$ & $86.5^{\mathrm{b}}$ & $71.5^{\mathrm{c}}$ & $P \leq 0.01$ \\
\hline 5 & Curvilinear velocity $(\mathrm{VCL})(\mathrm{m} / \mathrm{s})$ & $201.9^{a}$ & $194.8^{\mathrm{b}}$ & $152.1^{\mathrm{c}}$ & $P \leq 0.05$ \\
\hline 6 & Lateral amplitude of head displacement (ALH) (m) & $8.22^{\mathrm{a}}$ & $8.68^{\mathrm{a}}$ & $7.25^{\mathrm{b}}$ & $P \leq 0.01$ \\
\hline
\end{tabular}

Means bearing different superscripts in a row differ significantly, No. of Observations: 26

stages of cryopreservation cycle (Table-2).

The reduction in STR of swimming of bull spermatozoa from the initial to pre-freeze stage was significant $(\mathrm{P} \leq 0.05)$ but the post-thaw mean values did not show any significant difference over pre-freeze values (Table-2).

\section{Discussion}

The sperm motility (SM) of bull spermatozoa recorded in this study was in the same range $(66 \%$ to $96 \%$ ) of earlier reports [5,6]. Nevertheless, the PSM is marginally higher.

Comparing the results of other studies $[5,6]$, velocity traits of bull spermatozoa (VAP, VSL and VCL) seem to be highly variable and were around the mid-point of the range of values that were already observed. Usually factors such as age, time between ejaculations, degree of sperm maturation, energy stores (ATPase), the presence of surface-active agents in the cell membrane (agglutinins and detergents), viscosity of the fluids negotiated by the sperm, osmolarity, $\mathrm{pH}$, temperature, ionic composition of seminal plasma and possibly substances $(\mathrm{Cu}, \mathrm{Zn}, \mathrm{Mn}, \mathrm{Hg}$, hormones, kinins and prostaglandins) that stimulate or inhibit motility may affect sperm motility [7].

The CASA variables representing the head behaviour of motile spermatozoa (ALH (m) and BCF $(\mathrm{Hz})$ ) are also seem to be highly variable $[5,6]$. In this experiment the mean values for ALH and BCF are within the wide range cited by above authors. The swimming pattern of bull spermatozoa in terms of STR is similar to the earlier observations $[5,6]$.

Besides the biologic variability and differences between individual samples, the wide variation observed between many studies might be due to initial sampling of the biologic material, method of processing of semen for CASA, time elapsing between initial sampling and analysis, instrument settings and gates used in analyzing specimen, the accuracy of the specimen chambers used and the number of chambers, fields and sperm examined to provide adequate statistical sampling of the material analyzed [8].

In this experiment, a gradual significant decline in most of the sperm motion characteristics was noticed during the cryopreservation cycle, while the equilibration of semen before freezing has significantly reduced the proportion of motile sperm. This might be due to peroxidation of the fatty acids of the sperm, which destroys the structural integrity of plasma membrane leading to loss of motility $[9,10]$. Further, the exposure to the cryoprotective agent, glycerol in semen diluent is toxic to spermatozoa and brings reduction in sperm motility [11]. The path velocity, progressive velocity, beat cross frequency and straightness were also affected during the equilibration. Besides peroxidation and toxic effects of glycerol, the phospholipids of damaged spermatozoa produce the reactive oxygen species (ROS), which may be toxic to the normal spermatozoa [12,13], and in turn may contribute to reduction in sperm motion characteristics.

In this study, cryopreservation has drastically affected the quality of bull spermatozoa in terms of sperm motility, progressive sperm motility, path velocity, progressive velocity, curvilinear velocity, lateral amplitude of head and beat cross frequency. The progressive motility of spermatozoa is significantly affected by freezing process implies that the physiological basis for the progressive motility is very much sensitive to cryobiological damage [14]. Furthermore, changes in temperature impose changes on the composition and structure of various sperm plasma membrane domains [15]. Cryopreservation also induces apoptosis like changes [16] which may induce motility impairment. In addition, cryopreservation reduces the resistance of spermatozoa to ROS stress [17] and the levels of antioxidants [18], which may influence the sperm motion.

\section{Conclusion}

CASA technique enables objective evaluation motility characteristics of bull spermatozoa. Further, motion characteristics on velocity, head behaviour and 
swimming pattern of the spermatozoa can be assessed using CASA technique. The processing of semen namely equilibration, freezing and thawing significantly reduces the quality of semen in terms of almost all motion characteristics. Since CASA variables are known to be more useful in predicting the fertility of semen, adoption of CASA technique could help in better assessment of quality of frozen semen to improve fertility on AI. Nevertheless, further research on relating CASA variables with fertility would be useful for better utilization of CASA analysis.

\section{Author's contribution}

MNS conceived and designed the study, CASA analysis of semen, statistical analysis of the data, prepared and revised the manuscript. JK, evaluated, processed and cryopreserved the semen samples. KTPJ maintained the bulls and did the semen collections. All authors read and approved the final manuscript.

\section{Acknowledgements}

The authors acknowledge the Dean, Madras Veterinary College, Chennai - 600 007, India with thanks for the facilities provided for the study.

\section{Competing interests}

Authors declares that they have no competing interest.

\section{References}

1. Morris, G. J., Acton, E., Murray, J. B. and Fonseca, F. (2012). Freezing Injury; The special case of the sperm cell. Cryobiology, 64; 71-80.

2. Davis, O. R. and Siemers, J. (1995). Derivation and reliability of kinematic measures of sperm motion, Reprod Fertil Dev, 7; 857-869.

3. Forero-Gonzalez, R. A., Celeghini, E. C. C., Raphael, C. F., Andrade, A. F. C., Bressan, F. F. and Arruda, R. P. (2012). Effect of bovine sperm cryopreservation using different freezing techniques and cryoprotective agents on plasma, acrosomal and mitochondrial membranes. Andrologica, Doi: 111/j.1439-0272. 2010.01154.x.

4. Shiva Shankar Reddy, N., Jagan Mohan Rao, G. and Atreja, S. K. (2010). Effect of adding taurine and trehalose toa Tris based egg yolk extender on buffalo (Bubalus bubalis) sperm quality following cryopreservation. Anim. Reprod. Sci., 119; 183-190.

5. Farrell, B. P., Foote, R. H., Mcardle, M. M., TrouernTrend, L. V. and Tardif, A. L. (1996). Media human and dilution procedures tested to minimize handling effects on human, rabbit and bull sperm for computer assisted sperm analysis(CASA), JAndrol, 17;293-300.

6. Tardif, A. L., Farrell, P. B., Trouern-Trend, V. and Foote, R. H. (1997). Cmputer assisted sperm analysis for assessing initial semen quality and changes during storage at $5^{\circ} \mathrm{C}, \mathrm{J}$ Dairy Sci, 80; 1606-1612.
7. Blasco, L. (1984). Clinical tests of sperm fertilizing ability, Fertil Steril, 41; 177-192.

8. Farrell, B. P., Trouern-Trend, L. V., Foote, R. H. and Douglas-Hamilton, A. M. D. (1995). Repeatability of measurements on human, rabbit and bull sperm by computer assisted sperm analysis when comparing individual fields and means of 12 fields, Fertil Steril, 64, 208-210.

9. Kadirvel, G., Kumar, S. and Kumaresan, A. (2009). Lipid peroxidation, mitochondrial membrane potential and DNA integrity of spermatozoa in relation to intracellular reactive oxygen species in liquid and frozen-thawed buffalo semen. Anim. Reprod. Sci., 47; 143-150.

10. Waterhouse, K. E., Gjeldnes, A., Terdel, P. M., De Angelis, Farstad, W., Haard, M. and Kommiisrud, E. (2010). Alterations of sperm DNA integrity during cryopreservation procedure and invitro incubation of bull semen. Anim. Repro. Sci., 117; 34-42.

11. Maxwell, W. M. C. and Watson, P. F. (1996). Recent progress in the preservation of ram semen, Anim Reprod Sci, 42; 55-65.

12. Nichi, M., Goovaerts, I. G. F., Cortada, C. N. M., Barnabe. V. H., DeClercq, J. B. P. and Bolas, P. E. J. (2007). Roles of lipid peroxidation and cytoplasmic droplets in vitro fertilization capacity of sperm collected from bovine epididymides stored at 4 to $34^{\circ} \mathrm{C}$, Theriogenology, 67; 334-340.

13. Sariozkan, S., Bucak, M. N., Tuncer, P. B., Ulutas, P. A. and Bilgen, A. (2009). The influence of cysteine and taurine on microscopic oxidative stress parameters and fertilizing ability of bull semen following cryopreservation. Cryobiology, 58; 134-138.

14. Anel, L., de Paz, P., Alvarez, M., Chamorro, C. A., Boixo, J. C. (2003). Field and in vitro assay of three methods for freezing ram semen. Theriogenology, 60; 1293-1308.

15. Krogenaes, A., Anderson-Berg, K., Hafine, A. L. and Engeland, E. (1994). Membrane alterations in bull spermatozoa after freezing and thawing and after in vitro fertilization, Acta Vet Scand 35;17-26.

16. Kadirvel, G., Periasamy, S. and Kumar, S. (2012). Effect of cryopreservation on apoptotic-like events and its relationship with cryocapacitation of buffalo sperm. Reprod. Dom. Anim., 47; 143-150.

17. Nield, D. M., Brouwers, J. P., Colenbrander, B., Aguero, A., Gadella, B. M. (2005). Lipid peroxide formation in relation to membrane stability of fresh and frozen-thawed stallion spermatozoa, Mol Reprod Dev, 72; 230-238.

18. Bilodeau, J. F., Chatterjee, S., Sirard, M. A. and Gagnon, C. (2000). Levels of antioxidant defenses are decreased in bovine spermatozoa after a cycle of freezing and thawing, Mol Reprod Dev, 55;282-288.

$$
* * * * * * * *
$$

